\title{
Edme-François Jomard (1777-1862) et la mission égyptienne dans la première moitié du XIXe siècle
}

\section{Catherine Chadefaud}

\section{(2) OpenEdition \\ 12 Journals}

\section{Édition électronique}

URL : https://journals.openedition.org/dhfles/3409

DOI : $10.4000 /$ dhfles.3409

ISSN : 2221-4038

\section{Éditeur}

Société Internationale pour l'Histoire du Français Langue Étrangère ou Seconde

\section{Édition imprimée}

Date de publication : 1 décembre 2012

Pagination : 53-68

ISSN : 0992-7654

\section{Référence électronique}

Catherine Chadefaud, «Edme-François Jomard (1777-1862) et la mission égyptienne dans la première moitié du XIXe siècle ", Documents pour l'histoire du français langue étrangère ou seconde [En ligne], 49 | 2012, mis en ligne le 04 juillet 2016, consulté le 28 mai 2021. URL : http://journals.openedition.org/ dhfles/3409; DOI : https://doi.org/10.4000/dhfles.3409

Ce document a été généré automatiquement le 28 mai 2021.

(C) SIHFLES 


\title{
Edme-François Jomard (1777-1862) et la mission égyptienne ${ }^{1}$ dans la première moitié du XIXe siècle
}

\author{
Catherine Chadefaud
}

1 Dès avant l'expédition de Bonaparte en Égypte, l'élite française se passionne pour la découverte et la connaissance de l'Égypte ancienne. Divers récits de voyageurs alimentent des publications à succès : Volney a publié en 1784 le Voyage en Haute et Basse Égypte, Claude Savary relate en 1785 dans ses Lettres d'Égypte la visite de la grande pyramide de Chéops ${ }^{2}$.

2 Bonaparte s'inspire de l'idée de Talleyrand, ministre des Relations extérieures sous le Directoire. L'Expédition d'Égypte a pour objet d'affaiblir l'Angleterre en Méditerranée en lui barrant la partie terrestre de la route des Indes. La flotte quitte Toulon en mai 1798. Bonaparte dirige 32 généraux, 38000 soldats, 16000 matelots, et 187 érudits (les "savants "). Edme-François Jomard membre scientifique de l'expédition, en qualité d'ingénieur géographe eut un rôle déterminant dans les publications visant à faire connaître l'Égypte. Il prit en charge la direction de l'œuvre éditoriale officielle de la Description de l'Égypte. Sa passion pour ce pays et sa population l'amena à œuvrer pour appuyer la modernisation de l'état ottoman et de son élite.

\section{Le contexte: de l'Expédition d'Égypte (1798) à la fin de la Monarchie de Juillet (1848)}

\subsection{Jomard ingénieur géographe et éditeur scientifique}

3 Né en 1777 à Versailles, Jomard étudie à l'École des Ponts et chaussées puis à l'École polytechnique : en 1796 il devient Ingénieur-géographe du cadastre. Il participa à la totalité de l'expédition de 1798 à 1801 . Sur intervention de Monge et de Berthollet, qui en faisaient également partie, Jomard est nommé par Bonaparte membre de la Commission d'Égypte à partir de 1803, commission dont l'objectif est de réaliser et 
publier la Description de l'Égypte. Il devint, en 1807, secrétaire général de cette commission, puis en assure la coordination scientifique. La collecte des textes, des dessins et des cartes pour les planches est complexe. L'empereur exige un calendrier de publication. L'ensemble doit paraître entre 1807 et 1809 à une cadence semestrielle permettant de disposer simultanément des textes et des planches (in folio). Jomard dut surmonter les difficultés techniques pour sauvegarder la cohérence de l'ouvrage: translittération des caractères arabes ${ }^{4}$, échelles cartographiques, choix des prestataires pour la fourniture du papier, recherche de graveurs et d'aquafortistes. Jomard est aussi chargé des relations entre les quarante-trois auteurs. Après 1815, le gouvernement de la Restauration est favorable à l'achèvement du projet. Louis XVIII y accorde intérêt et bienveillance.

4 En 1818 Jomard est élu membre de l'Académie des inscriptions et Belles Lettres. En 1821, il est l'un des fondateurs de la Société de Géographie à Paris ${ }^{5}$.

\subsection{Jomard et les expériences antérieures dans l'enseignement et la pédagogie.}

5 En juin 1815, Jomard fait un séjour professionnel en Angleterre pour compléter les vérifications de certaines planches dessinées de la Description de l'Égypte. À Londres, Jomard s'intéresse aux écoles d'apprentissage mutuel ${ }^{6}$. À son retour à Paris, il participe à la fondation de la "Société pour l'instruction élémentaire $»^{7}$ et peu après il devient chef du Bureau d'instruction publique à la préfecture de la Seine ${ }^{8}$. Cette pédagogie de l'enseignement élémentaire pour laquelle Jomard a tant œuvré connaît en France un certain succès en $1820^{\circ}$. Dans ce type d'enseignement mutuel la pédagogie est mise en œuvre par des laïcs ${ }^{10}$.

\subsection{L'espoir de Jomard s'affirme : propager les idées des Lumières au pays de Méhémet Ali.}

6 Quel est le contexte de la naissance de L'École égyptienne en France dont Jomard prit l'initiative? Entre 1803 et 1811 Jomard n'a jamais cessé d'entretenir des relations épistolaires avec des personnalités égyptiennes. Il reste attentif aux questions de géographie, de cadastre, d'agronomie et de modernisation du pays. En 1809 Méhémet Ali est devenu pacha de la province turque d'Égypte, il revendique en 1815 l'indépendance de cette vaste province face au sultan Mahmoud (Tulard 1983 : 40-41). Dès 1811 Jomard écrit un Mémoire au consul français d'Alexandrie Bernardino Drovetti, d'origine piémontaise; ce document, destiné au pacha, préconise de faire venir en France de jeunes Égyptiens pour les former, les instruire dans divers métiers utiles pour moderniser leur pays.

7 Le pacha hésite d'autant plus que les usages traditionnels de l'époque favorisent plutôt la présence des Italiens et leur coopération diplomatique et commerciale à Alexandrie. Dès 1817 le pacha avait fait appel à des Français pour moderniser le pays ${ }^{11}$ et en 1824 il avait sollicité le gouvernement français pour former une mission qui pourrait le conseiller sur des réformes militaires : le général Belliard avait été envoyé en Égypte à cet effet ${ }^{12}$.

8 La période n'est guère favorable, car en France c'est le mouvement philhellène qui est au premier plan dans la diplomatie : la défense de la Grèce opprimée par les Ottomans. 
À Paris au Salon de peinture, Delacroix présente le tableau «Les massacres de Scio " (1824) puis l'allégorie «La Grèce sur les ruines de Missolonghi » (1826).

Drovetti, consul de France en Égypte ${ }^{13}$ demeure le meilleur informateur de Jomard à partir de 1819. Méhémet Ali avait envoyé à Paris un diplomate Hadji Osman-Noureddin pour solliciter la formation de jeunes élèves égyptiens aux sciences et aux arts. Les conflits de la Morée retardèrent ce projet. Drovetti finit par peser sur la décision de Méhémet Ali en 1825 d'envoyer de jeunes Égyptiens (une quarantaine) pour se former en France ${ }^{14}$. Entre temps Jomard avait aussi entretenu des relations épistolaires avec un diplomate Félix Mengin qui avait publié en 1823 un ouvrage relatif aux modernisations de l'Égypte selon la volonté de Méhémet Ali (Mengin 1823) ${ }^{15}$. Et en avril 1826 le nouveau consul de France au Caire, Malivoire, instruit le ministre des Affaires Étrangères de Charles $\mathrm{X}$ de la décision du pacha.

Les jeunes gens qui forment cette mission égyptienne sont âgés de dix-sept à vingtquatre ans, issus d'excellentes familles (turques, circassiennes, géorgiennes, arméniennes...). Ils sont conduits et encadrés par trois responsables (ou effendis) ${ }^{16}$ et par un jeune imam, Rifa'a al-Tahtawi, ancien étudiant de la mosquée El-Azhar chargé de veiller sur l'intégrité religieuse de ses compagnons. Cette mission égyptienne embarque à Alexandrie en mars 1826 sur un vaisseau de guerre français et débarque à Marseille. Selon l'usage pour l'arrivée d'étrangers venant d'Orient, les jeunes gens subissent la quarantaine au lazaret du lieu.

11 Jomard, à Paris, est alors prévenu qu'il est désigné par le gouvernement comme directeur des études ${ }^{17}$. Le contexte n'étant guère favorable aux membres de l'empire ottoman en général, Jomard conseille que les jeunes gens revêtent un costume occidental avant de partir pour Paris et de s'y installer ${ }^{18}$.

\section{La mission égyptienne voulue par Méhémet Ali et la naissance de l'École franco-égyptienne 1826/1827}

\subsection{Jomard organisateur}

12 Les étudiants et leurs accompagnateurs furent logés d'abord à Clichy puis à Paris (rue du Regard, près du quartier Latin). Pendant la première année, les élèves acquièrent des notions de français et des rudiments de mathématiques, dont les résultats sont évalués chaque mois. Ils sont ensuite intégrés aux cours élémentaires des collèges royaux par petits groupes. Pour contrôler le niveau acquis, Jomard organise un examen sanctionné par une remise de prix dont Méhémet Ali est informé par courrier ${ }^{19}$. Satisfait, il propose à Jomard un traitement annuel que ce dernier refuse! Jomard affirme :

13 Je n'ai consenti à diriger les études des jeunes Égyptiens qu'avec l'approbation d'un grand nombre de personnes amies des Lumières et de l'humanité, et à la condition que mes travaux seraient gratuits [...]. En instruisant ces jeunes étrangers, nous formons autant d'amis à la France, autant d'appréciateurs de notre état social; et un jour nos voyageurs, nos artistes et nos commerçants, appelés sur les bords du Nil, trouveront en eux un appui assuré et l'affection de la reconnaissance ${ }^{20}$. 


\subsection{Examen et distribution des prix $(1827 / 1828)$} milieu de la France, puisez à pleine source les lumières de la raison et des lettres, qui élèvent si haut l'Europe au-dessus des autres parties du monde. C'est reconquérir pour votre patrie les bienfaits des lois et des arts, dont elle a joui tant de siècles; l'Égypte, dont vous êtes les députés, ne fait, pour ainsi dire, que recouvrer ce qui lui appartient, et la France, en vous instruisant, ne fait qu'acquitter, pour sa part, la dette contractée par toute l'Europe envers les peuples de l'Orient (Jomard 1828b, cité dans Laissus 2004 : $322)$.

\subsection{Le devenir de l'École égyptienne dans un contexte difficile (1830 à 1835)}

18 Un suivi pédagogique individuel est mis en place, sans que les détails en soient connus. La synthèse est transmise à Méhémet Ali qui assume les frais des études des jeunes gens. L'entreprise de Jomard reste cependant confrontée à un contexte difficile. Il n'est pas appuyé par le ministère de Charles $\mathrm{X}$, en raison des évènements qui sont une source de conflits avec la Sublime Porte: la Grèce, soutenue par la France et l'Angleterre obtient son indépendance par le traité d'Andrinople en 1829. Les prétentions algériennes de la France ouvrent une autre crise majeure en juin 1830. Puis éclate, la même année, la Révolution de juillet qui aboutit à la prise de pouvoir de Louis-Philippe Ier. Jomard demande audience au nouveau ministre Guizot. Il sollicite de nouvelles autorisations administratives et de l'aide pour l'arrivée d'autres étudiants égyptiens à Marseille et leur installation à Paris (Dossier Jomard: Lettre n' 104, 11 août 1830). C'est une nouvelle promotion qui arrive pour un cycle d'études de trois ans que Jomard voudrait pouvoir prolonger jusqu'à cinq $\mathrm{ans}^{25}$. 
19 En 1832, on note que 28 des 42 étudiants venus en France en 1826 sont repartis en Égypte une fois leurs études terminées, tandis que 34 nouveaux étudiants sont arrivés. Jomard fait à la Société de Géographie à Paris divers comptes rendus sur le flux régulier des élèves égyptiens venant étudier en France ${ }^{26}$. L'un des premiers étudiants à repartir après un examen satisfaisant en 1830 est Tahtawi. Il s'est spécialisé dans les travaux de traduction et il a remis à un jury de deux orientalistes (Sylvestre de Sacy et Caussin de Perceval) un manuscrit sur sa relation de voyage en France. En Égypte, Tahtawi devint professeur de langue française à l'école de médecine d'Abou-Zabel, fondée par Clot-Bey près du Caire ${ }^{27}$. D'autres étudiants repartirent après une formation dans la marine de guerre française, d'autres ont bien réussi dans la formation agricole à Roville ${ }^{28}$.

En 1832 ils sont au total 80 en formation dans diverses spécialités. Arrivent à Paris douze élèves médecins avec leur maître le docteur Clot-Bey (Hamont 1843, II : 188), médecin français au service du gouvernement égyptien, fondateur de l'école de médecine du Caire. Un jury de la faculté de médecine les répartit pour compléter leur formation dans les hôpitaux parisiens (Val de Grâce, Hôtel Dieu et La Charité). Après seize mois ils passent avec succès leurs examens. Jomard s'efforce de faire connaître cette réussite à travers divers articles ${ }^{29}$.

\subsection{L'évolution des relations diplomatiques et le devenir de l'École égyptienne (1835/1839).}

Hélas les relations diplomatiques du gouvernement de Louis Philippe avec celui de Méhémet Ali se dégradent et le ministre Boghos-Bey annonce en décembre 1835 que Méhémet Ali ordonne le retour au Caire de tous les étudiants égyptiens. Un prétexte est évoqué : la peste vient de sévir en Égypte. Bon nombre d'enseignants sont décédés et le pacha a un besoin urgent de personnel formé (Laissus 2004 : 329-330). Faut-il voir dans la décision brutale du pacha quelque déception ou plus probablement, l'influence de la diplomatie anglaise hostile à tout rôle français en Égypte?

Jomard ne s'avoue pas vaincu et présente en avril 1836 à l'Académie des inscriptions et Belles-Lettres une publication d'une soixantaine de pages: Coup d'œil impartial sur l'état présent de l'Égypte, comparé à la situation antérieure. Dans ce document, Jomard compare la situation de l'Égypte en 1798, 1821 et 1833 et défend l'œuvre accomplie par Méhémet Ali. Jomard aborde aussi le projet de percement de l'isthme de Suez ${ }^{30}$ et les effets bénéfiques qui pourraient rejaillir sur le commerce égyptien (Laissus 2004 : 557). À la même période huit jeunes égyptiens arrivent en France pour étudier. Jomard les prend en charge toujours avec zèle et soutient les efforts de ces huit derniers étudiants en médecine et en chirurgie.

Les affaires d'Orient sont complexes : la question de l'accès des détroits est débattue par les puissances européennes et le tsar de Russie veut intervenir contre la Sublime Porte. Méhémet Ali entend conquérir définitivement son indépendance par rapport à l'empire Ottoman. Mais en raison de la négociation engagée sur les détroits, il ne peut compter sur l'appui des nations européennes. En 1839 Jomard n'hésite pas à prendre à nouveau la défense de Méhémet Ali en France en participant à la rédaction de l'ouvrage de Félix Mengin (1839) (cf. Laissus 2004 : 559-560). Il a perdu son meilleur informateur Drovetti (rentré en France). L'armée du jeune fils de Méhémet Ali vient d'écraser en Syrie l'armée turque. Le sultan Mahmoud II meurt: complots et ambitions désorganisent l'empire ottoman. Après diverses péripéties, Méhémet Ali doit renoncer 
à la Syrie mais il conserve la vice-royauté d'égypte établie à titre héréditaire dans sa famille. Pendant cette longue crise, Jomard affirme : «Rien ne me fera renoncer aux idées d'avenir pour l'Égypte ».

\section{De Jomard expert sur « l'Orient » à Jomard-Bey}

\subsection{Jomard expert consulté.}

Dans les années qui suivent 1839, Jomard continue à être une sorte d'expert pour tout ce qui touche à l'Égypte : il reçoit et conseille le ministre égyptien des travaux publics en 1844. Il continue à espérer et à s'informer sur la situation en Égypte et fait connaître à Paris la réussite professionnelle des anciens étudiants venus en France.

Hamont, membre de l'école vétérinaire d'Alfort, vient d'enseigner deux ans en Égypte, à la demande de Méhémet Ali. À son retour il publie un ouvrage en 1843 et donne un aperçu des réalisations et des perspectives ouvertes par le séjour des jeunes égyptiens en France depuis 1826 (Hamont 1843, II : 181 sq.), tout en soulignant les limites liées au fait qu'ils s'étaient rarement vu attribuer des postes de premier plan dans l'administration.

\subsection{Suivre les nouvelles venant des anciens étudiants.}

Dans la dernière phase de sa vie, Jomard apprend avec joie par l'un de ses anciens étudiants que le pacha (devenu vice-roi) a chargé Mouktar-Bey, autre ancien étudiant devenu président du conseil de l'instruction publique, d'organiser au Caire une école d'administration civile. Elle inscrit dans ses rangs tous les anciens étudiants venus en France. Ce projet se concrétise après 1837. Jomard est convaincu que les réformes les plus urgentes concernent l'agriculture et le statut des terres, et principalement les mesures pour favoriser l'irrigation. Tahtawi réalise son plus bel espoir. Il obtient en Égypte la création en 1835 d'une école de traduction: une équipe de spécialistes commence à traduire en arabe bon nombre d'ouvrages occidentaux utiles dans un État qui se modernise. Jomard dans une lettre de janvier 1838 (Dossier Jomard: Lettre $\mathrm{n}^{\circ}$ 135) fait état du dynamisme de cette école de traduction où plusieurs traités d'agronomie sont traduits en arabe.

Cependant l'enthousiasme de Jomard est parfois teinté d'inquiétude sur les méthodes de gouvernement et les objectifs réels poursuivis par le pacha ${ }^{31}$. L'auteur s'en ouvre à Prosper Enfantin en 1844, il écrit :

Faut-il faire un crime à Méhémet-Ali d'avoir gouverné avec fermeté ? Je n'ose dire. Il faut tout placer dans la balance. Il faut tenir compte de la condition commune à tout musulman (...) J'ai inculqué à mes cent vingt pupilles égyptiens, autant que j'ai pu, quelque chose de plus que les principes des arts, que les éléments des sciences, c'est le sentiment de la justice et celui des droits de l'humanité. Ces germes ne périront point, pas plus que ceux que j'ai contribué, vingt-cinq ans avant à faire croître aux rives du Nil : grâce au ciel, ils ont porté leur fruit (Fonds Enfantin, Ms 7735, pièce 14).

Jomard tente de se rassurer sur le bien-fondé de son entreprise et se prend à espérer la naissance d'une élite cultivée et occidentalisée mais dans un futur lointain. Hamont (1843, II : 190-195) note que la formation des élites est encore précaire. Dans l'immédiat, l'objectif des réformes du pacha demeurait l'art de la guerre ${ }^{32}$. Jomard avait-il rêvé au 
long de ces années d'un Orient sensible à l'Europe des Lumières, s'était-il bercé d'une illusion? (Bret $2003: 5$ sq., 14).

\subsection{Jomard reçoit le titre honorifique de Bey en 1856 : de Méhémet Ali à Saïd quels changements ?}

En 1846 Ibrahim, fils et héritier de Méhémet Ali est reçu à Paris ; peu après, est fondée à Paris la «Société d'études du canal de Suez» (cf. Mantran 1989, chap. XI) et Jomard devient président de la Société de Géographie (1848). Méhémet Ali meurt en 1849. Son petit-fils Abbas lui succède : l'attitude anti-européenne de ce dernier afflige Jomard ${ }^{33}$. Mais en 1856 Saïd qui a succédé à Abbas mène une politique différente vis à vis des Européens. Il renoue avec Jomard dont il fait un correspondant scientifique, et l'élève à la dignité de Bey. En 1858 a lieu la Première réunion du Conseil d'administration de la Compagnie universelle du canal de Suez. Jomard est l'un des trois présidents honoraires.

Jomard meurt à Paris dans la nuit du 23 septembre 1862 à sa table de travail. Ainsi disparaît le dernier des érudits de l'expédition d'Égypte. La décoration de la Medjidié décernée par l'administration égyptienne à la demande de Saïd arrive après sa mort et a été remise à ses enfants. Tout au long de cette expérience d'enseignement voulue par Jomard, il est permis de se demander quelles furent les intentions réelles de Méhémet Ali au delà du discours diplomatique. Jomard héritier des Lumières se lança dans ce projet avec l'espoir de créer une élite francophone au sein de la société égyptienne. Son projet connut une réussite limitée en raison des intentions non avouées de l'entourage de Méhémet Ali et des pressions qui s'exercèrent sur l'Égypte de la part de l'empire ottoman, de l'empire russe et de l'empire britannique. Pour assurer la construction de l'état égyptien et se détacher de l'empire ottoman, Méhémet Ali pratiqua plusieurs politiques en parallèle. L'une consistait à flatter les intentions et l'idéal français de l'époque des Lumières, l'autre consistait à chercher des hommes compétents pour préparer la modernisation de l'armement et trouver des financements pour lutter contre la volonté de reprise de contrôle de l'État ottoman. L'empire russe supportait mal l'idée d'une présence étrangère autre que la sienne auprès du Vice-roi d'Égypte, soucieux qu'il est de contrôler au plus près sa politique d'affaiblissement de l'empire ottoman. La question du contrôle des détroits était déjà à l'ordre du jour. Quant à l'empire britannique, sa politique de contrôle de la route des Indes par la voie terrestre lui faisait craindre toute influence autre que la sienne en Égypte. L'État français supporta les efforts de Jomard avec la prudence que lui inspirait l'hostilité des autres acteurs internationaux. Puis les relations de la France et de l'Égypte prirent une nouvelle tournure à la suite de l'acceptation du projet du canal de Suez par le Vice-roi d'Égypte. Les questions d'éducation des personnels égyptiens ne furent plus de mise dans les échanges diplomatiques entre l'État français et le Vice-roi. L'École francoégyptienne ne présentait plus d'intérêt pour le gouvernement français. Seule persista du projet de Jomard l'École de traduction fondée par Tathawi en Égypte. 


\section{BIBLIOGRAPHIE}

\section{Sources}

CORRESPONDANCE de François Grille (1728-1855), Ms 624. Bibliothèque municipale d'Angers.

DOSSIER JOMARD, Panthéon versaillais. Bibliothèque municipale de Versailles.

FIERRO, Alfred. (1984). Inventaire des manuscrits de la Société de Géographie. Paris : BNF.

FONDS ENFANTIN, Ms. 7735. Bibliothèque de l'Arsenal.

JOMARD, Edme-François (dir.) (1808-1828). Description de l'Égypte. 9 vol. texte in-folio et 14 volumes de planches grand in-folio. Paris : Imprimerie Nationale.

JOMARD, Edme-François. (1816). « Rapport général sur la situation des écoles établies d'après le principe de l'enseignement mutuel dans les départements, dans la capitale et aux environs, suivi d'un extrait de la correspondance étrangère, lu par M. Jomard, l'un des secrétaires de la Société (pour l'enseignement élémentaire), à l'assemblée générale du 23 août 1816 ». Journal d'Éducation publié par la Société formée à Paris pour l'amélioration de l'enseignement élémentaire, t. II, 11, 275-298.

JOMARD Edme-François. (1828 a). « De jeunes Égyptiens envoyés à Paris en 1826 ». Le Moniteur, mercredi 12 mars 1828, 299. Revue Encyclopédique, t. XXXVII, 843-845.

JOMARD, Edme-François. (1828 b) « École égyptienne de Paris ». Nouveau journal Asiatique, t. II, 96-116

JOMARD, Edme-François (1836). « Lettre de M. Jomard à M. le président de la Société de Géographie. Paris, le Ier décembre 1836 (pour faire hommage d'un recueil de dissertations universitaires rédigées par de jeunes Égyptiens ayant étudié à Paris) ». Bulletin de la Société de Géographie, 2e série, t. IX, janvier 1838, 53.

JOMARD, Edme-François (1840). «Égypte. Canaux d'irrigation ». Bulletin de la Société de Géographie, 2e série, t. XIV, 5-9.

LAISSUS, Yves (2004). Jomard, le dernier égyptien. Paris : Fayard.

MENGIN, Félix (1839). Histoire sommaire de l'Égypte sous le gouvernement de Mohammed-Aly de l'an 1828 à l'an 1838. Paris : Firmin Didot.

\section{Études}

ALEXANDRE-BIDON, Danièle, COMPERE, Marie-Madeleine, GAULUPEAU, Yves et collab. (1999). Le patrimoine de l'éducation nationale, Charenton-le-pont : Flohic éditions.

BERCHET, Jean-Claude (1985). Le voyage en Orient. Anthologie des voyageurs français dans le Levant. Paris : Laffont.

BRET, Patrice (1998). La vie quotidienne en Égypte au temps de Bonaparte. Paris : Hachette.

BRET, Patrice (2003). «L'Égypte de Jomard. La construction d'un mythe orientaliste, de Bonaparte à Méhémet Ali ». Romantisme, revue des études romantiques et dix-neuvièmiste, 33e année, 120, 2e trimestre, 5-14.

CARRÉ, Jean-Marie (1956). Voyageurs et écrivains français en Égypte, vol. I. Le Caire : IFAO.

DOUIN, Georges (1923). Une mission militaire française auprès de Mohammed-Aly

(correspondance des généraux Belliard et Boyer). Le Caire : IFAO. 
DROVETTI, Bernardino (2003). Lettres de B. Drovetti, consul de France à Alexandrie (1803-1830) présentées et commentées par Sylvie Guichard. Paris : Maisonneuve Larose.

FIERRO, Alfred (1983). La Société de Géographie (1821-1946). Paris : Champion.

GOBY, Jean-Edouard (1987). Dictionnaire Napoléon sous la direction de Jean Tulard, (article « Jomard »). Paris : Fayard.

HAMONT, Pierre N. (1843). L’Égypte sous Méhémet Ali. 2 vol., Paris : Léautey \& Lecointe.

LAISSUS, Yves (1998). L'Égypte, une aventure savante (1798-1801). Paris : Fayard.

LAISSUS, Yves (2004). Jomard, le dernier Égyptien. Paris : Fayard.

LAURENS, Henry, Gillispie, Charles, Golvin, Jean-Claude, Traunecker, Claude (1997) [1989].

L’Expédition d'Égypte 1798-1801. Paris : Colin.

LOUCA, Anouar (1970). Voyageurs et écrivains égyptiens en France au XIXe siècle. Paris : Didier.

MANTRAN, Robert (dir.) (2005) [1989]. Histoire de l'empire Ottoman, Paris : Fayard.

PANZAC, Daniel \& RAYMOND, André, (2002). La France et l'Égypte à l'époque des vice-rois (1805-1822). Cahier des Annales islamologiques, 22.

TAHTAWI, Rifa'a al (1988). L'Or de Paris, traduit et présenté par Anouar Louca. Paris : Sindbad.

TULARD, Jean (1983). « Bonaparte en Égypte », L’Histoire, 61, 30-41.

ZEND, Pierre (1974) « La méthode pédagogique de Jean-Baptiste de La Salle au début du XIXe siècle ». Histoire de l'enseignement de 1610 à nos jours, (Actes du 95ème Congrès national des sociétés savantes, Reims, 1970), t. I. Paris : CTHS, 61-81.

\section{NOTES}

1. La mission égyptienne est le terme officiel donné par Méhémet Ali et les ministres français à l'entreprise que les contemporains appellent École franco-égyptienne.

2. A propos des divers voyageurs $c f$. Carré (1956, II) et Berchet (1985). Vivant Denon qui participa à l'expédition publia à son retour un ouvrage intitulé Voyage dans la Basse et Haute Égypte pendant les campagnes du général Bonaparte.

3. Sur l'ensemble de l'expédition se reporter à Laurens, Gillispie, Golvin, Traunecker, (1989) avec bibliographie et cartographie. Pour un exposé rapide et synthétique incluant le rôle de Méhémet Ali cf. Tulard (1983: 40-41).

4. Les travaux relatifs au monde ottoman furent confiés à Jacques Joseph Marcel, arabisant, qui avait été responsable de l'imprimerie en langue arabe lors de l'expédition en 1798. Rentré à Paris il devint à partir de 1803 Directeur de l'Imprimerie nationale et créa le département chargé des transcriptions de l'arabe dans les volumes de la Description de l'Égypte.

5. Sur la carrière de Jomard pendant l'expédition de Bonaparte (cf. Carré 1956 : chap. III) et pour la participation à la publication de la Description de l'Égyptecf. Goby (1987 : 973). Sur son rôle à la Société de Géographie voir Fierro (1983).

6. Méthodes de Bell et d'autre part de Lancaster. Bell, pasteur anglican propose dès 1797 une méthode de mutual tuition : des élèves moniteurs enseignent à de plus jeunes selon les conseils et les préceptes d'un maître. Dans le même esprit Lancaster développe un monitorial system dans un quartier populaire de Londres.

7. Il rejoint Lasteyrie, de Gérando et Laborde. Sur les méthodes et les réalisations de l'enseignement mutuel en France et le monitorat voir Alexandre-Bidon (1999 : 348-353).

8. Il occupa ce poste jusqu'en 1823 . 
9. D'autres petites écoles de ce type ont été fondées à Paris et dans plusieurs villes de France. L'une des caractéristiques de la pédagogie consiste à enseigner la lecture et l'écriture de front tandis que dans les Écoles des Frères (dans la tradition de Jean-Baptiste de la Salle) la lecture doit être maîtrisée avant qu'on enseigne l'écriture. Dans l'enseignement mutuel les rudiments de l'arithmétique sont complétés par du dessin linéaire. Pour les filles, des notions de couture sont proposées. Cette pédagogie nouvelle fut parfois introduite dans des entreprises auprès des apprentis.

10. Après un réel succès, ce type d'enseignement connut un ralentissement après 1830 et rencontra aussi l'opposition du clergé catholique ainsi que d'autres pédagogues (cf. Zend 1974 : 61 sq.)

11. Alexis Jumel dans le domaine du textile et du développement de la culture du coton, Pascal Coste sur recommandation de Jomard et J. Anthelme Sève afin de réformer l'armée, puis en 1819 Antoine Clot chirurgien venu de Marseille. Sur le contexte de cette période voir Panzac et Raymond (2002).

12. Belliard à la tête d'une dizaine de personnes demeura à Alexandrie pendant deux ans pour évaluer la nature des demandes du Pacha (cf. Douin 1923 et Laissus 2004 : 307).

13. Drovetti (1776-1852) participa à l'expédition d'Égypte en 1798. Devenu par la suite consul de France au Caire il profite de ce statut privilégié pour chercher et acheter des objets de fouilles. Il vend ensuite sa collection personnelle au roi de Piémont-Sardaigne qui l'installe en son palais de Turin. Jomard correspondait avec Drovetti de façon régulière depuis 1819 (cf. Guichard 2003 : Lettres).

14. Drovetti a réussi à vaincre l'influence italienne et anglaise auprès de Méhémet Ali. (Louca $1970: 33 \mathrm{sq})$.

15. Jomard avait contribué à la rédaction d'une longue annexe de 66 pages sur le contexte géographique et historique des espaces gouvernés par le pacha (Laissus $2004: 547, n^{\circ} 126$. Cette numérotation a été établie par Laissus pour classer les sources des écrits de Jomard).

16. Il s'agit d'Abdi Effendi en qualité de garde des Sceaux (« Muturdar »), de Mustafa Mukhtar en qualité de responsable des finances («Defterdar») et de Hassan Effendi (Jomard 1828 t. II : 109 sq).

17. Il est assisté d'un inspecteur des études, le cairote Joseph Agoub, professeur d'arabe à Louis le Grand à Paris et qui enseigne le français et la littérature (Bret 2003 7sq).

18. Dossier Jomard : lettre $n^{\circ} 67,25$ mai 1826. Dans ce message Jomard s'inquiète des dépenses de loyers à prévoir en indiquant que la durée des études pourra s'étendre sur 3 à 5 ans.

19. L'intention de Méhémet Ali est d'établir une fondation pour ces jeunes élèves (Dossier Jomard : Lettre $n^{\circ} 68,26$ mai 1826).

20. Extrait de la réponse de Jomard publiée dans le Moniteur du 12 mars 1828 (Laissus 2004 : 319 et note 1$)$.

21. Les spécialités sont: 1 . administration civile, 2 . administration militaire, 3 . navigation et marine, 4. diplomatie, 5. hydraulique, 6. mécanique, 7. génie militaire, 8. artillerie, 9. fonderie des métaux et fabrication d'armes, 10. gravure, typographie et lithographie, 11. arts chimiques, 12. médecine, chirurgie, anatomie, physiologie et hygiène, 13. agriculture, 14. histoire naturelle et mines, 15. traduction. Pour des indications détaillées sur la répartition de ces enseignements et sur le corps professoral consulter Hamont (1843, II : 184-188).

22. Mathieu de Dombasle (né en 1777) a rédigé des articles sur ses innovations rurales et techniques de 1824 à 1837 dans les Annales agricoles de Roville.

23. Hamont (1843, II : 186-188) indique qu'il tient ses sources d'un compte-rendu de Jomard luimême. Les jeunes étudiants ont entre dix-sept ans et trente-sept ans. Cependant un jeune élève en gravure et lithographie a quinze ans et un autre en sciences naturelles a seize ans. 
24. L'examen écrit comporte une composition française, une analyse logique et grammaticale, et un problème de mathématiques. Pour l'épreuve de dessin on compare les dernières réalisations des élèves ( $c f$. le texte de Jomard dans le Nouveau Journal Asiatique).

25. Dans cette même lettre, Jomard sollicite de pouvoir venir présenter à Guizot M. le Muhurdar effendi, chef de la mission égyptienne avec plusieurs des travaux des jeunes étrangers. Il précise que la mission égyptienne se compose alors de 80 élèves.

26. Jomard tente aussi d'obtenir des aides financières de la Société de Géographie mais en vain.

27. Tahtawi vécut jusqu'en 1873. Antoine Clot-Bey né à Marseille, vécut jusqu'en 1868.

28. Les élèves ayant fait un cycle à Roville sont pris en charge, à la demande de Jomard, par Pierre Thomas Marie qui leur enseigne l'arpentage sur le terrain (Dossier Jomard : Lettre $n^{\circ} 106$, septembre 1831).

29. Et aussi par des images: une lithographie de Charles Bazin en 1831 représente Jomard entouré de jeunes étudiants égyptiens (Laissus $2004: 519, \mathrm{n}^{\circ} 4$ ).

30. Ce sujet avait déjà fait l'objet des réflexions des saint-simoniens depuis 1833 et l'arrivée au Caire de quelques représentants dont Enfantin et les ingénieurs Ch. Lambert et H. Fournel ( $c f$. Carré, I, 264 sq.).

31. Jomard essuie diverses critiques dont celle de Chateaubriand en 1848 dans les Mémoires d'Outre-tombe à propos de l'évolution de l'Orient turc (livre dix-neuvième, chapitre 15).

32. Sur les avis très partagés quant aux réformes de Méhémet Ali et à la modernisation du pays voir Carré (1956, I, 279-297). Prisse d'Avesnes qui séjourné longtemps en Égypte donne aussi un avis défavorable.

33. Abbas (règne de 1849 à 1854) avait désapprouvé la politique de Méhémet Aliet il cherche à détruire son œuvre et toute l'influence européenne : il ferme l'École polytechnique du Caire, il ferme le bureau de traduction dirigé par Tahtawi, de même que l'École de médecine de Clot-Bey qui se résout à rentrer en France. Des travaux de barrage sur le Nil confiés à un ingénieur français sont suspendus.

\section{RÉSUMÉS}

En 1801, au retour de l'expédition d'Egypte, Edme-François Jomard ingénieur géographe qui avait fait partie des civils du corps expéditionnaire garda des liens avec des lettrés arabes du Caire et des diplomates français en Orient. Il obtint de Méhémet Ali, pacha, la création d'une «mission égyptienne » à Paris. Malgré un contexte international tendu, ce projet prit la forme d'une école destinée à former en France une élite d'administrateurs, d'ingénieurs et d'agronomes pour moderniser l'état égyptien et qui recevra chaque année plusieurs dizaines d'étudiants. Cette expérience échoue après la mort de Méhémet Ali (1849) ; seule une école de traduction organisée au Caire par Tahtawi, ancien élève à Paris lui survit.

After 1801, during the return journey from Egypt, E.-F. Jomard, geographical engineer who had been member of the Civil Corps, stayed in touch with Arabian scholars and with French diplomats in Egypt. He built under the authority of the Pacha Mohammad Ali, a training school project for administrators, engineers and agronomists able to modernize Egyptian state. In spite of a stressed international context, the school operated every year with several tenths of students. The Egyptian School in Paris failed after Mohammad Ali's death (in 1849); a translation school organized in Cairo by Tahtawi, ancient student in Paris, will survive. 
INDEX

Mots-clés : campagne d'Égypte (1798), école franco-égyptienne / Mission égyptienne, E.F.

Jomard, Méhémet Ali, Tahtawi (Rifa'a-al)

Keywords : Franco-Egyptian School/Egyptian mission, French Expedition in Egypt (1798, E.-F.

Jomard, Mohammad Ali, Tahtawi (Rifa'a-al)

\section{AUTEUR}

CATHERINE CHADEFAUD

cchadefaud@wanadoo.fr 